\title{
TRAICIÓN EN LA TRADUCCIÓN LITERARIA - ¿ERROR COMÚN O SACRIFICIO PRODUCTIVO?
}

\author{
Kurt Rüdinger \\ Universidad de Sevilla
}

\begin{abstract}
The following article is an attempt of response to ancient critics about translation of fictional texts. The first part will prove that treason is an unavoidable companion of translation in general terms and naturally even more, if the original text is an artistic product. The second part deals about the necessity of changing the point of view: If treason seems unavoidable, we shouldn't claim for tricks to suppress it, but look for some productive integration in translator's and critic's work. For that reason we will claim for some new criterions of analyse and application. The third part will exemplifie how these criterions may work on theoretical and practical duties.
\end{abstract}

\section{NOCIONES PREVIAS}

Es un más que conocido tópico que traducción es traición, y más cuando se trata del "arte cuya herramienta es la palabra". Cabe recordar al respecto el famoso ensayo "Miseria y Esplendor de la Traducción" de Ortega y Gasset. Sin embargo, la polémica es más antigua que aquel trabajo de 1937. Sin exageración se puede decir que el reproche tiene tanta tradición como la traducción misma.

Esa mala fama que lleva la traducción desde sus inicios se ve sorprendentemente contrastada con un consumo más que abundante de los frutos de semejante labor tan tramposa. Sin detallar hechos históricos podemos destacar que la actividad traductora empezó a prosperar con la aparición de tecnologías de divulgación masiva de textos escritos (¡Gutenberg!) y la creciente alfabetización de capas sociales hasta entonces excluídas de aquel logro civilizatorio. Aunque los mismos autores en gran medida hayan protagonizado la cruzada contra los traidores de sus biensonantes y refinados pensamientos, hay que destacar que desde la Odisea de Homero hasta el Werther de Goethe el patrimonio de la literatura mundial se deletrearía de otra forma, si estas obras se hubiesen percibido únicamente en sus respectivos idiomas. Es más, que se sepa, ningún Premio Nobel de las últimas decadas habría conseguido esta distinción, sin que se hubiese traducido su obra a una multitud de idiomas extranjeros. Además, una fugitiva mirada hacia las listas de bestseller en Alemania y España nos enseña que las plazas privilegiadas no las ocupa ningún autor autóctono en ambos casos, sino uno estadounidense, con los títulos traducidos, desde luego.

Aunque sí es cierto, que un hecho no se autoconsagra a través de su mera existencia, hay que preguntarse cómo una actividad supuestamente tan 'delictiva' puede tener tanta acogida al mismo tiempo ${ }^{1}$.

\footnotetext{
${ }^{1}$ La analogía con el narcotráfico - que también goza de lucros inmensos y mala prensa a la vez - no conviene, suponiendo que en este caso las críticas se lanzan desde una parte que no está implicada en el asunto, mientras que en el mundo literario son los mismos protagonistas del ámbito lingüístico-literario que calientan la polémica
} 
A pesar de las críticas es evidente que haya algunas aportaciones innegables de aquel tramposo oficio: la intermediación cultural a gran escala es impensable si tenemos en cuenta que hasta en las culturas más 'abiertas' como mucho se llega a un bilingüismo a nivel social (ej.: Escandinavia con inglés como L2 profundizada y generalizada).

En lo siguiente analizaremos en un primer paso algunos de los reproches más comunes mediante citas de algunos críticos destacados para contrastar los argumentos aducidos con una serie de tópicos fundamentales de lingüística general y teoría de comunicación. De esta forma intentaremos llegar a unos conceptos más sobrios, respectivamente una fundamentación teórica de estas críticas. Sin entrar aquí en detalles, adelantamos que la traición en la traducción literaria se plasmará como condición indispensable de la misma y no como obstáculo esquivable de mayor o menor medida, según que caso concreto.

La segunda parte asumirá este resultado para plantear una nueva visión de la traducción con el intento de darle una función productiva a la traición en su ámbito, si ya parece inevitable la convivencia con ella.

La tercera parte se dedicará a la aplicación de los criterios esbozados en la segunda. Veremos como esa nueva visión obra tanto en la evaluación crítica de traducciones hechas como en la labor práctica en cuanto se trate de tomar decisiones concretas y a veces incisivas.

\section{OPINIONES DESTACADAS ACERCA DE LA (IN)TRADUCTIBILIDAD DE TEXTOS LITERARIOS Y SU TRANSCENDENCIA TEÓRICA}

\subsection{Apología y objeciones}

Propondremos en primer lugar una cita apológica acerca de la traductibilidad para que sirva de ejemplo de un desmesurado optimismo traductológico que de forma casi natural provoca las críticas que a continuación se citarán:

Die Sprachen sind ein Mittel, dadurch die Menschen einander ihre Gedancken offenbaren können: Da nun die Gegenstände, womit die Menschen sich in ihren Gedanken beschäftigen, überhaupt in der ganzen Welt einerley und einander gleich sind; da die Wahrheit, welche sie mit dieser Beschäftigung suchen, nur von einer Art ist; und da die Gemüthes-Kräfte der Menschen auf eine gleiche Art eingeschränket sind; so muß notwendig unter den Gedancken der Menschen ziemliche Gleichgültigkeit statt und platz haben; daher denn solche auch in dem Ausdrucke nothwendig wird. - Auf diesem Grunde beruhet nun die ganze Kunst, aus einer Sprache in die andere zu übersetzen. Von einem Übersetzer wird erfodert ( $¡$ sic!), da $\beta$ er eben dieselben Begriffe und Gedancken, die er in einem trefflichen Muster vor sich findet, in eben solcher Ordnung, Verbindung, Zusammenhange, und mit gleich so starckem Nachdrucke mit andern gleichgültigen bey einem Volck angenommenen, gebräuchlichen und bekannten Zeichen ausdrücke, so daß die Vorstellung der 
Gedanken unter beyderley Zeichen einen gleichen Eindruck auf das Gemüthe des Lesers mache. (Breitinger, 1966: 138).

Cabe destacar que esta postura hace un flaco favor al conjunto de traductores, poniéndoles el listón muy alto. La suposición que toda la humanidad piense igual y por tanto sea susceptible a las mismas emociones que sólo requieren un estímulo análogo es más que dudosa de por sí. La convicción de que existen estos estímulos análogos en cualquier idioma es simple secuela de la primera idea y el imperativo categórico de que el traductor los busque tanto le pone en apuros en su trabajo como lo hace excesivamente vulnerable respecto a la crítica de su labor. Desde luego, esta visión optimista de la traducción no quedó sin respuestas. Veremos algunas a continuación:

Alles Übersetzen scheint mir schlechterdings ein Versuch zur Auflösung einer unmöglichen Aufgabe. Denn jeder Übersetzer muß immer an einer der beiden Klippen scheitern, sich entweder auf Kosten des Geschmacks und der Sprache seiner Nation zu genau an sein Original oder auf Kosten seines Originals zu sehr an die Eigentümlichkeiten seiner Nation halten. Das Mittel hierzwischen ist nicht bloß schwer, sondern geradezu unmöglich. (Humboldt, 1970: 144).

Es evidente que, donde Breitinger (1966) proclama la indivisión absoluta de la humanidad Humboldt aboga por el polo opuesto: cada cultura está enjaulada en su idiosincrasia de una manera que considera imposible establecer un puente lingüístico viable entre una y otra. Conclusión única:

qué el traductor -al menos él de literatura- se busque otro oficio, siendo improcedente el que se propone.

No obstante, ambas posturas tienen en común que no se incomodan mucho en buscar los argumentos a su favor en criterios propios de los sistemas lingüísticos cuya (in)intermediabilidad proclaman. Observemos a continación dos visiones algo más afinadas al respecto:

Dichtung ist unübersetzbar. Ihr Klang ist unübersetzbar, ihr Rhythmus, ihre Melodie, aber das ist es nicht allein. Dichtung ist unübersetzbar, weil sie uns auffordert, nicht nur durch die Sprache hindurch, über die Sprache hinaus, sondern auch auf die Sprache selbst zu blicken. Dichtung ist die große andere Möglichkeit der Sprache, die Möglichkeit, das Werkzeug zum Kunstwerk zu machen. (Wandruszka, 1967: 7).

El avance respecto a los anteriormente citados es, sin duda, la división de lengua como medio de comunicación por un lado y como fuente de arte por el otro. El efecto desmitificador en cuanto al lenguaje común, referencial nos ayudará en el enfoque del 
lenguaje artístico como objeto discreto de observación, es decir, la pormenorización de aspectos propios de la traducción literaria.

En la misma línea, más breve, pero ya enfocada en un aspecto muy concreto por considerar, se puede leer la siguiente cita (Bloomfield, 1935: 278): "as to denotation, whatever can be said in one language can doubtless be said in any other: the difference will concern only the structure of the forms, and their connotation.

Si entendemos la postura de Wandruszka como visión general e idealizada de lengua como arte, Bloomfield destaca dos aspectos particulares que pueden influir "técnicamente" en la traductibilidad de un texto literario: el de la semántica de un lenguaje que transgrede lo puramente referencial, considerando connotaciones idiomáticas, y el de la diferencia en la estructura de formas, supuestamente de suma importancia a la hora de traducir poesía.

Resumiendo lo arriba expuesto, se pone de relieve que al menos las tres primeras argumentaciones se apoyan de sin intermediación en conceptos metafísicos, Weltgeist en Breitinger, idiosincrasia cultural en Humboldt, lenguaje autointrospectivo en Wandruszka que, aunque acertaran parcial- o completamente, aportan poco al análisis y a la práctica en casos concretos, ya que siguiendo sus pasos nos quedarían tan sólo dos opciones: mejor ni empezar (Humboldt, Wandruszka) o desesperar ante un reto sobreelevado (Breitinger).

Sentimos por tanto la necesidad de valernos con algunos argumentos lingüísticos fundamentales que nos den una orientación más sobria sobre lo que hay y lo que no puede haber en nuestro ámbito en cuestión.

\subsection{Lingüística general/teoría de comunicación y traduccion literaria}

Aunque el siguiente pequeño repaso parezca escueto, teniendo en cuenta las numerosas y alargadas exposiciones traductológicas, estamos convencidos de que la reflexión sobre dos conceptos fundamentales de los planteamientos de Saussure y de Bühler y su confrontación con las objeciones principales arriba mencionadas nos desvelarán exhaustivamente la magnitud de la 'miseria de la traducción'.

Veamos. Si bien se sabe que el Curso de Lingüística General de Ferdinand de Saussure es algo como el grito inicial de la Lingüística como ciencia propia, dejando atrás primeros intentos de los neogramáticos y positivistas que sólo trataban la parte material, es decir, fonética de la lengua, y más atrás aun los conceptos idealistas de "almas bellas" en el sentido de Schiller, como fue por ejemplo el mismo Humboldt. Si este último plasma la lengua como unidad indisoluble entre forma y contenido -idea que, desde luego proclama la inintermediable idiomaticidad de cada lengua natural- la novedad introducida por Saussure son las famosas dos caras de la moneda del signo lingüístico, es decir, la división en significante y significado: 
El lazo que une el significante al significado es arbitrario, o también, ya que por signo entendemos la totalidad resultante de la asociación de un significante a un significado, podemos decir más sencillamente: el signo lingüístico es arbitrario.

Así la idea de "sœur" '[hermana] no está ligada por ninguna relación interior con la serie de sonidos $s-\ddot{o}-r$ que le sirve de significante; también podría estar representada por cualquier otra: prueba de ello: las diferencias entre las lenguas y la existencia misma de lenguas diferentes: el significado "bœuf" tiene por significante $b-\ddot{o}-f$ a un lado y $o-k-s$ (Ochs) al otro. (Saussure, 1991: 104).

Arbitrario es todo, así entendemos a Saussure en este apartado: el conjunto de sonidos que aprueba una comunidad lingüística como significante, el concepto o significado al que sirve como tal, y, aunque no se haya mencionado literalmente, se supone que el alcance semántico de dicho significado también. Sin embargo, a este nivel no se ve ningún obstáculo para una posible traducción, siempre que rija la conciencia de distintos marcos semánticos. Si pensamos p.ej. en una encimera de material duro con tres o cuatro patas, vulgo "mesa" en castellano, no habrá grandes objeciones contra una adaptación por Tisch en alemán o table en inglés, maniobra que sin falta concederían Bloomfield y Wandruszka, pero quizás no Humboldt.

Por la esbozada arbitrariedad del signo lingüístico tampoco pasa nada, si el profesional dedicado a la producción de semejante mueble está derivado del objeto o no : Tischler vs. carpintero vs. carpenter y todavía no pasa nada, si queremos traducir frases referenciales como El carpintero produce una mesa vs. Der Tischler stellt einen Tisch her. Hasta aquí ni Bloomfield ni Wandruszka tendrían inconvenientes, pero Humboldt ya con toda seguridad.

No obstante, la cosa se complica, cuando a un autor alemán se le ocurre el capricho estilístico:

Der Tischler tischlert einen Tisch quizás reciclado en un pareado como Der Tischler tischlert einen Tisch - Der Fischer fischt sich einen Fisch, etc. En este caso la traición parece inevitable.

En resumen, tal como es correcta la división entre significado y significante aplicada al lenguaje común, en 'el arte cuya herramienta es la palabra' el significante pierde su cómoda condición de arbitrario sea solamente en el sentido fonético y/o sea respecto al concepto al que sirve como tal y está comprometido de por sí, para no decir significativo.

En esta circunstancia, según nuestro entender, hay una reconciliación inesperada entre los conceptos de Humboldt y Saussure sin que ninguno de los dos tuviera que renunciar a la válidez de sus criterios.

Con otro tópico saussureano nos acercamos al problema planteado por Bloomfield, hablamos del valor lingüístico de un signo en un idioma determinado que, según Saussure se define a través de lo que otros no son, en otras palabras, a base de la particular ocupación de un campo semántico: 
Si las palabras se encargaran de representar conceptos dados de antemano, de una lengua a otra tendrían correspondencias exactas para el sentido; pero no es así. El francés dice indistintamente louer [alquilar] (una casa) para "tomar" o "dar en alquiler", allí donde el alemán emplea dos términos: mieten y vermieten; no hay pues correspondencia exacta de valores: Los verbos schätzen y urteilen presentan un conjunto de significaciones que corresponden, en general, a las de las palabras francesas estimer y juger; sin embargo, esa correspondencia falla en muchos puntos. (Saussure 1991: 165).

En conclusión, los valores y con ellos, por supuesto, las posibles connotaciones difieren de un idioma a otro de forma incalculable.

Recordemos por otro lado a Karl Bühler y su conocido Organonmodell de la comunicación humana ${ }^{2}$. Se supone que un mismo mensaje albergue con mayor o menor intensidad las tres funciones: referencial, apelativa y expresiva. No obstante, cierta explicitud sólo requiere la primera de ellas, las otras dos pueden esconderse más o menos detrás de lo referencial y también con distinta evidencia en diferentes idiomas. Por ejemplo, en alemán, pronunciado con determinado énfasis, la frase "es zieht!" implica sin más "mir ist kalt!" (función expresiva) y al mismo tiempo "mach's Fenster zu!" (función apelativa) al menos esta última no la vemos con la misma claridad en la versión española del referente "¡Sopla aire!”.

Parece que el planteamiento de Bühler, relacionado con Bloomfield en este caso, también condena la posibilidad de traducción literaria al fracaso, y eso sin que tuviese que forzar argumentos metafísicas de incompaginabilidad.

Para concluir esta pequeña sinopsis lingüística llegamos al deprimente resultado que por la propia naturaleza de lengua, respectivamente las lenguas en cuestión, la traducción literaria está entregado a la traición por sistema. Veremos a continuación qué horizontes se le ofrecen en estas circunstancias.

\section{ESBOZO DE NUEVAS PAUTAS EN CRÍTICA Y PRÁCTICA DE LA TRADUCCIÓN LITERARIA A BASE DE LA INEVITABILIDAD DE TRAICIÓN}

Tal como resaltó en el capítulo anterior, la traducción y la traición no mantienen una simple relación del tipo 'objetivo - obstáculo' que con más o menos habilidad puede solucionarse favorablemente, sino que la traición se plantea como condición indispensable de la traducción sobre todo en su faceta literaria. Reconociendo que sea así, no obstante, nos vemos sorprendidos por una avalancha de literatura traductológica que no se cansa en emitir un sinfín de criterios, consejos, técnicas y trucos de cómo evitar o al menos atenuar el peligro de la traición en este ámbito, sin dar abasto ni avanzar, desde luego, y, lo que tampoco extraña mucho, empezando cada vez de nuevo y de cero, fenómeno que se refleja

\footnotetext{
${ }^{2}$ Véase la breve presentación del concepto en: Hadumod Bußmann, Lexikon der Sprachwissenschaft, Stuttgart, Kröner, 1983, p. 365.
} 
en la abundante frecuencia de vocablos como introducción y Einfïhrung en los títulos de semejantes publicaciones. De forma que, a pesar de tanto empeño, las traducciones siguen siendo 'malas' y 'tramposas' tanto en la opinión de los críticos y muchos autores -sin renunciar a lucro y prestigio que les facilitan los resultados- como en la de los hipotéticos o reales usuarios de las mismas y, para más inri, en la de más de un traductor, al menos en cuanto se trate de la labor de otros representantes de su profesión.

Para poner fin a debates tan infinitos como desagradables nosotros nos adheriremos a la postura que José Ortega y Gasset calificó como la del buen optimista:

El buen optimista, en cambio, piensa que puesto que sería deseable libertar a los hombres de la distancia impuesta por las lenguas, no hay probabilidad de que se pueda conseguir; por tanto, que sólo cabe lograrlo en medida aproximada. Pero esta aproximación puede ser mayor o menor..., hasta el infinito, y ello abre ante nuestro esfurzo una acyuación sin límites en que siempre cabe mejora, superación, perfeccionamiento; en suma: <progreso>. (Ortega y Gasset, 1987: 26).

En estas líneas destaca el mismo concepto de imperfección inesquivable como la decisión de darle una vuelta productiva en vez de disuasiva a esta condición.

Nuestra propuesta aboga entonces por aceptar el que "cuando traduzco traiciono" y por guiar las decisiones concretas a base de esta conciencia, en vez de llevar una lucha inútil contra lo inevitable. Para que no haya confusiones. Eso no quiere decir que reclamemos un catch as catch can salvaje, ni un descarado Ist der Ruf erst ruiniert, lebt sich's völlig ungeniert (ambas posturas recabadas en el concepto de Ortega y Gasset sobre el mal optimista), pero la ética subyacente se deletrea de forma distinta.

Bajo el lema "traducción/traición", según nuestro entender, hemos de distinguir en primer plano entre traiciones útiles y traiciones improcedentes.

Estos conceptos, desde luego, requieren una explicación. $\mathrm{Ni}$ en el mundo del que se deriva la palabra, es decir, el ámbito de la delincuencia, se traiciona por mero gusto, sino para conseguir algo. Si aplicamos este móvil a nuestro tema,la traducción literaria, podemos concluir que, para que una traición tenga sentido, tiene que recompensarnos con algún logro que de otra manera no se hace asequible. Más concreto, será provechoso sacrificar una forma estricta (esquema de rima, ritmo, etc.), si de tal modo nos aproximamos al contenido del original. Viceversa podemos permitirnos ciertos 'arreglos semánticos', si consideramos esencial que se mantengan ciertos rasgos formales (véanse ejemplos más adelante).

Traiciones improcedentes consideramos todas aquellas que se desvían sin recompensa por otra parte. Podrían clasificarse también como fallos, pero hay que tener cuidado, ya que probablemente no se deben tanto a la incompetencia o incomprensión del traductor, sino a una decisión suya poco afortunada. En los textos que veremos más adelante habrá ejemplos de ambas facetas -error propiamente dicho y traición improcedente. 
A estas alturas vemos tres opciones fundamentales entre las que puede elegir un traductor/traidor de cara a un texto concreto:

La primera -quizás la más cómoda, pero también la más dolorosa- es la renuncia. Si un texto no promete ninguna aportación a L2 y su cultura, sea por contenido o sea por un sobrepeso de determinados rasgos de su significante -ipara qué traducirlo? La respuesta nos resulta fácil, si vemos poesías como Ottos Mops kotzt de Ernst Jandl - ¡para nada!

A la segunda opción nos gustaría llamarla traición del arte, para caracterizar de tal manera cualquier traducción que se centra de forma unilateral o casi unilateral en el aspecto informativo, referencial del texto y no se preocupa mucho de cuestiones etilísticas ni formales. Esta clase de traducción suele ser poco atrayente en el sentido estético, por lo cual su sitio lógico no estará precisamente en las listas de bestseller, sino más bien en ámbitos didácticos como veremos en el ejemplo de Rilke más abajo, o bien para dar una información semántica sobre un producto que conviene ser consumido en su versión original (p.ej. canciones).

La tercera opción la llamaremos traición con arte. En este concepto vemos recabado cualquier intento de transmitir a L2 la condición artística, respectivamente estética del original. Según lo arriba expuesto es evidente que en este empeño aceche la traición en cada detalle. Sin embargo, parece también la única manera de conseguir como producto algo que se lea con gusto parecido al que damos por supuesto en caso del original, sea éste el que fuere.

Conclusión: Según los criterios arriba plasmados distinguimos dos tipos fundamentales de traición en la traducción literaria:

- la traición de arte

- la traición con arte

Dentro de cada tipo identificamos tres clases de 'infracción':

- la traición útil

- la traición improcedente

- el fallo común

A continuación aplicaremos este instrumentario mínimo a una serie de ejemplos como esbozo de evaluación, en cuanto se trate de traducciones hechas y publicadas y como autoevaluación en caso de que se trate de borradores inéditos, susceptibles aun de mejora.

\section{APLICACIÓN DE LOS CONCEPTOS ESBOZADOS A EVALUACIÓN Y AUTOEVALUACIÓN}

Tal como anunciado estudiaremos los diferentes conceptos de traición en su aplicación a casos concretos. Si en lo siguiente, salvo en un ejemplo, nos reduciremos exclusivamente al género lírico será fundamentalmente por dos razones relacionadas entre sí: el lenguaje 
poético, así se supone, es el más idiosincrático por antonomasia, de forma que la aptitud de los criterios propuestos se evidencia con más claridad que en la mayoría de los textos de prosa, lo cual también se debe al hecho de que las obras mismas se presentan más cortas, concentradas y así presentables en su totalidad.

El primer ejemplo lo elegimos por parecer un caso típico de traición de arte. Veremos original y traducción en el contraste:

Rainer Maria Rilke

Der Panther

Im Jardin des Plantes, Paris

Sein Blick ist vom Vorübergehen der Stäbe so müd geworden, dass er nichts mehr hält.

Ihm ist als ob es tausend Stäbe gäbe und hinter tausend Stäben keine Welt.

Der weiche Gang geschmeidig starker Schritte, der sich im allerkleinsten Kreise dreht, ist wie ein Tanz von Kraft um eine Mitte, in der betäubt ein grosser Wille steht.

Nur manchmal schiebt der Vorhang der Pupille sich lautlos auf-. Dann geht ein Bild hinein, geht durch der Glieder angespannte Stille und hört im Herzen auf zu sein.

versus

E. Barjau

La pantera

En el Jardin des Plantes, París

Cansada del pasar de los barrotes, su mirada ya no retiene nada.

es igual que si hubiera mil barrotes y detrás de ellos no quedara mundo.

Su blando andar, de fuertes pasos ágiles, dando vueltas en círculo minúsculo, es una danza de una fuerza en torno a un centro donde, artudido, se alza un gran deseo.

Sólo, a veces, se apartan las cortinas de la pupila, sin ruido: una imagen cruza 
la tensa calma de sus miembros, y allá en su corazón deja de ser. ${ }^{3}$

Comentario breve: en la traducción no se observa ningún intento de atender a alguno de los rasgos estilísticos o formales del original, pero tampoco destacan traiciones improcedentes ni fallos, en resumen un ejemplo logrado de traición de arte. De tal modo cumple con su función primordial dentro del planteamiento de la obra en la que se publica, suponiendo que prevalece en ésta el objetivo didáctico.

El segundo ejemplo, el único del género prosa, lo hemos enfocado respecto a un recurso particular de traición de arte y por cometer un error grave al llevarlo a cabo. Veremos el ejemplo, para cuya claridad no hace falta citar el original:

Luego silbó una canción. Había estado ensayando desde otoño. Sabía tocar una serie de canciones. Sah ein Knab y Ein Schifflein sah ich fahren, Kapitän und Leutenant

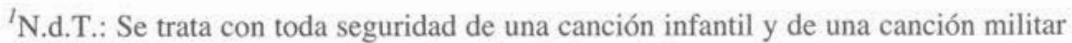
popular, respectivamente. La traducción de los títulos sería: "Vio un niño" y "Un barco vi pasar, capitán y teniente." ${ }^{4}$

Comentario breve: la nota a pie de página es una intervención "traidora" por antonomasia porque interrumpe la integridad del discurso literario. A veces será imprescindible para intermediar entre algo idiomático-intraducible y la consiguiente incomprensión del lector hipotético, pero, desde luego, tiene que acertar. Desafortunadamente esto no es el caso en nuestro ejemplo. Sah ein Knab - "Vio un niño" de ningún modo es una canción infantil, sino el primer verso del Heideröslein de J.W. von Goethe, una poesía de tan reconocida connotación erótica que en una ocasión ha llegado al tan cuestionable como significativo honor de subtitular una de las típicas secuencias de fotos de la edición alemana del Playboy. En cambio, la música con la que esa poesía se ha reciclado como canción es de un tal Franz Schubert, máximo exponente del género Kunstlied - entre todo un fallo imperdonable por parte del traductor.

Si los ejemplos arriba expuestos los consideramos traiciones del arte, veremos a continuación dos trabajos que por intención, quizás no por logro, se clasificarían como traiciones con arte, es decir que se empeñan en rescatar el carácter artístico del original. El primer ejemplo en esta linea es la traducción del famoso soneto A una nariz de Quevedo hecha por Wilhelm Muster. Comparemos:

\footnotetext{
${ }^{3}$ Textos tomado de La Literatura Alemana a través de sus textos, L. Acosta (1997: 796).

${ }^{4}$ Trad. de "Sie sehen den Marmor nicht" de Ernst Schnabel a cargo de Antonio J. Fernández, No Ven el Mármol, en Hermes 1, Sevilla, 1993, p. 75.
} 
Francisco de Quevedo

A una nariz

Érase un hombre a una nariz pegado, érase una nariz superlativa, érase una naríz sayón y escriba, érase un peje espada muy barbado.

Era un reloj de sol más encarado, érase una alquitara pensativa, érase un elefante boca arriba, era Ovidio Nasón más narizado.

Érase un espolón de una galera, érase una pirámide de Egipto, las doce tribus de narices era.

Érase un naricísimo infinito, muchísima nariz, nariz tan fiera, que en la cara de Anás fuera delito.

versus

Wilhelm Muster

Auf eine Nase

Es war ein Mann, der hing an einer Nase, es war die Nase superlativiert.

Es war ein kleines Ding an einer Nase!

Es war Ovidios Naso, höchst nasiert,

es war die Sonnenuhr mit imposantem

Zeiger, ein Schwertfisch, sehr benast, und war gereckt der Rüssel eines Elefanten und zwölfer Judenstämme Nasenschar.

Es war ein Kolben, eines Schiffes Schnabel! Es war der Pharisäer Nasennase, dem Hohenpriester Annas wär' sie leid!

Es war einmal ein Nasenturm zu Babel, der endlos langen Dinge Paraphrase 
und nasnasierte Nasenewigkeit. $^{5}$

Propuesta de evaluación escueta sin entrar en todos los detalles:

De la forma del poema se guarda el género de soneto, pero con otro esquema métrico. Por las conocidas dificultades de mantener un estricto "abba abba cdc dcd" románico, parece un sacrificio defendible para conseguir la forma lírica en alemán.

Respecto al contenido observamos que Wilhelm Muster refleja el argumento principal 'descripción de una nariz descomunal'. Se puede admitir el cambio de orden de algunas imágenes por razones métricas y para que sea biensonante. No obstante, es dudosa la necesidad de supresión, respectivamente sustitución de algunas (véanse los versos 3, 10, 13 en el original). Según nuestro entender el traductor se ha liberado indebidamente del original, es decir, su labor es antes Nachdichtung que traducción, en nuestros términos un caso de traición improcedente.

El siguiente ejemplo es de nuestro propio taller y nos servirá aquí junto con una propuesta de autoevaluación como el concepto de traición con arte puede guiar el trabajo práctico. Si la calificación resulta demasiado positiva se debe al carácter subjetivo que semejantes procedimientos nunca pueden suprimir por completo. Veamos el Soneto de repente de Lope de Vega el correspondiente intento de traición con arte:

\author{
Soneto de repente \\ Un soneto me manda hacer Violante, \\ que en mi vida me he visto en tanto aprieto; \\ catorce versos dicen que es soneto: \\ burla burlando van los tres delante. \\ Yo pensé que no hallara consonante \\ y estoy a la mitad de otro cuarteto, \\ mas si me veo en el primer terceto, \\ no hay cosa en los cuartetos que me espante. \\ Por el primer terceto voy entrando, \\ y parece que entré con pie derecho, \\ pues fin con este verso le voy dando. \\ Ya estoy en el segundo, $\mathrm{y}$ aun sospecho \\ que voy los trece versos acabando; \\ contad si son catorce, $y$ está hecho.
}

\footnotetext{
${ }^{5}$ Francisco de Quevedo, Gedichte Spanisch und Deutsch - Ubertragung und Nachwort von Wilhelm Muster mit zwölf Illustrationen von Hans Fronius Cotta, Stuttgart, J.G. Cotta'sche Buchhandlung Nachfolger GmbH, 1982, p. 32-35.
} 
versus

Sonett aus dem Stande

Ein Sonett zu schreiben, hieß mich die Violant', nie je in meinem Leben mußt ich mich so eilen..

Ein Sonett, so sagt man, zählt vierzehn Zeilen:

Spöttisch spöttelnd (Mir nichts dir nichts) stehen deren drei vorderhand.

Mir deuchte, als fände ich keinen Konsonant, und mitten im nächsten Quartett wir schon weilen. Doch seh' ich am ersten Terzett mich feilen, in den Quartetten kein Grund zur Sorge bestand.

Ins erste Terzett werd' ich mich nun begeben; mit dem rechten Fuß, so scheint's, ich Zutritt erlang'. Durch diesen Vers will ein End' ich ihm geben.

Schon bin ich im zweiten und noch ist mir bang, zu beenden die dreizehn ist derweil mein Bestreben; Zählt ruhig, es sind (ob es) vierzehn, und das Werklein gelang.

Propuesta de autoevaluación:

Se respetan al máximo género y esquema métrico, salvo leves diferencias de ritmo y intonación.

Se transmite el argumento principal. "El soneto contempla su propia ontogenia."

En la relectura nos pareció más apropiado mir nichts dir nichts en vez de spöttisch spöttelnd por ser más idiomático y transparente en alemán.

El cambio de persona en el segundo verso del segundo cuarteto se debe a razones formales, es decir conservación del esquema métrico. De todos modos no nos pareció un sacrificio semántico grave, dado que el soneto invita a todos (¿nosotros - los lectores?) a ver su propio desarrollo.

Para el último verso ofrecemos como alternativa compatible con el resto. Zählt ruhig, ob es vierzehn, und das Werklein gelang por captar la idea del original de forma más literal.

\section{CONCLUSIÓN}

Los criterios arriba establecidos, desde luego, aún son rudimentarios. Sin embargo, creemos que admiten la integración de cualquier aspecto habitual de análisis e interpretación de textos bajo su lema.

La ventaja que vemos, si aceptamos la traición como fiel acompañante de cada traducción literaria, es doble: 
- En la evaluación/crítica de traducciones hechas se impone un filtro que canaliza las objeciones y en cierto modo pone las riendas a exigencias desmesuradas que anublan con frecuencia el reconocimiento de verdaderos logros de una propuesta de traducción. La idea básica en la que se apoyan evaluaciones convencionales que "dos cosas distintas nunca serán lo mismo", en cambio, aporta poco en su simple apodicticidad.

- El traductor obtiene un marco orientativo sencillo a través de cual puede medir y autoevaluar inmediatamente los pros y contras de sus decisiones sin repasar interminables catálogos de buenos consejos y advertencias, siendo eso una actividad de la que dudamos que alguien la efectúe, por cierto.

Interesante sería para el futuro estudiar la aplicabilidad de los conceptos esbozados a textos de prosa, donde vemos como reto primordial la definición de lo artístico en el original para derivar a partir de ello las conclusiones críticas y prácticas respecto a la traducción.

\section{REFERENCIAS BIBLIOGRAFICAS}

ACOSTA, L. A. (coord.), La Literatura Alemana a través de sus textos, Madrid, Cátedra, 1997.

BLOOMFIELD, L., Language, Londres (Nueva York), George Allen and Unwin, 1935.

BREITINGER, J.J., Critische Dichtkunst, 1740, Stuttgart, Deutsche Neudrucke. Reihe Texte des 18. Jahrhunderts, vol.2, 1966.

BUSSMANN, H., Lexikon der Sprachwissenschaft, Stuttgart, Kröner, 1983.

FERNÁNDEZ, A. J., No Ven el Mármol, trad. de Ernst Schnabel, "Sie sehen den Marmor nicht", en Hermes, 1, Sevilla, 1993.

HUMBOLDT, W., "Brief an A.W. Schlegel vom 23. Juli 1796", en:. Hartmann, P. I Vernay, H. (eds.), Sprachwissenschaft und Übersetzen, Múnich (Commentationes Societatis Linguisticae Europaeae, 3), 1970.

KOLLER, W., Einführung in die Übersetzungswissenschaft, 4ªed., Heidelberg, UTB, 1992.

ORTEGA Y GASSET, J., Miseria y Esplendor de la Traducción, München, dtv 9123, 1987.

QUEVEDO, F., Gedichte Spanisch und Deutsch - Übertragung und Nachwort von Wilhelm Muster mit zwölf Illustrationen von Hans Fronius Cotta, Stuttgart, J.G. Cotta'sche Buchhandlung Nachfolger GmbH, 1982.

SAUSSURE, F., Curso de Lingüística General, trad. por Mauro Armiño, Madrid, Akal, 1991.

WANDRUSZKA, M., "Die maschinelle Übersetzung und die Dichtung”, Poetica, 1, 1967. 